\title{
Slow Relaxation of Resistance in Manganite Perovskite Nanoconstrictions
}

\author{
J. BASZYŃSKI \\ Institute of Molecular Physics, Polish Academy of Sciences \\ M. Smoluchowskiego 17, 60-179 Poznań, Poland
}

\begin{abstract}
The time decay of the surface resistance $R_{\mathrm{S}}(t)$ was measured for the $\mathrm{La}_{0.7} \mathrm{Sr}_{0.3} \mathrm{MnO}_{3}$ perovskite nanocontacts obtained by break technique at liquid nitrogen. The corresponding time relaxation can be described by two-term formula: stretched exponential function and logarithmic dependence. The characteristic times $\tau_{1}$ and $\tau_{2}$ of the relaxation processes are of order of seconds and few tens of seconds, respectively. The appearance of both contributions evidences the existence of two sources of relaxation, which can be assigned to inhomogeneous changes of the angle between the magnetic moments of the neighboring $\mathrm{Mn}$ ions inside spin blocks $\left(\tau_{1}\right)$ and between the spin blocks $\left(\tau_{2}\right)$ occurring near surface of the nano-electrodes. Similar to magnetic viscosity, the dependence of resistance viscosity $S_{\mathrm{R}}$ on basis current is characterized by bell-shaped curve. These results point out the important role of the structural arrangements of the Mn-ions into spin blocks on the surfaces electrodes of the nanocontact.
\end{abstract}

PACS numbers: 75.60.Lr, 75.50.Pp

\section{Introduction}

Ferromagnetic, half-metallic rare-earth manganite perovskites show colossal magnetoresistance (CMR) and spin polarization making them promising candidates for an emerging oxide spin-electronics (see Ref. [1] and references therein, [2]). There are two main areas for spintronic applications of these materials: first as the sources of spin polarized materials in multilayered structures for spintronics applications and second as nanoconstriction of sensors. The problems of the magnetism of the nanoconstrictions, i.e., the surface magnetic properties, have attracted the attention of scientists for many decades. The control of these properties is one of the most important issues in the development of spintronics. A knowledge of surface properties is essential not only for the development of a perovskite manganite-based technology but also for determination of the fundamental phenomena and mechanisms of magnetoelectronic behavior. The generally accepted Zener double exchange model [3], which is commonly used for a description of manganese compounds, assumes that the magnetic field aligns the local Mn- $t_{2 \mathrm{~g}}$ spins ferromagnetically and facilitates hopping of the conduction electrons Mn- $e_{\mathrm{g}}$ between adjacent $\mathrm{Mn}$-ions sites. Due to this close relation between transport properties and the magnetization in manganese compounds [4], the resistance measurements provide an excellent indirect method for characterization of the magnetic relaxation observed in atomic scale constrictions. Resistance relaxation in $\mathrm{La}_{2 / 3} \mathrm{Sr}_{1 / 3} \mathrm{MnO}_{3+\delta}$ (LCMO) films was first investigated by van Helmolt et al. [5] and the results were discussed in terms of spin-glass properties and magnetic viscosity. The electronic structure of the surface layer implies that exchange is anisotropic, i.e., the exchange stiffness is different on a path within the layer compared to a path perpendicular to it. The surface magnetic and electronic properties are of paramount importance for spin injection. It is well known that the surface composition of thin films differs from bulk and in the consequence the magnetism of the surface is significantly different from that of the bulk [6]. The preparation procedure determines the nature of the surface and this plays a very crucial role in electrical transport and magnetic properties. Contrary to film structures, in our experiment the surface composition of the nanoconstriction electrodes obtained by break technique is supposed to be bulk like. In this paper, we report the relaxation of surface resistance $R_{\mathrm{S}}$ of the $\mathrm{La}_{0.7} \mathrm{Sr}_{0.3} \mathrm{MnO}_{3}$ electrodes observed within the time window $0.2 \mathrm{~s}<t<100 \mathrm{~s}[7,8]$.

\section{Results and discussion}

The perovskite nanocontacts (PNC) of this study were prepared by breaking technique from $\mathrm{La}_{0.7} \mathrm{Sr}_{0.3} \mathrm{MnO}_{3}$ ceramics at liquid nitrogen (LN) [7, 8]. Due to the use of breaking technique the clean surfaces of the electrodes of the nanocontacts were obtained whose compositions are identical to that of the bulk ceramics of perovskite. A smooth and continuous variation from point contacts to a vacuum barrier tunnel regime is observed when mechanical contact of the electrodes is reduced. The strong 
freezing of Mn-ions spins due to random exchange interactions or random anisotropies at the surface cause such a remarkable relaxation of the surface resistance in nanocontact of the perovskites. To our knowledge, our experiment is the first of this type measurements where the relaxation surface resistance $R_{\mathrm{S}}$ is examined using the circular magnetic field $H_{\text {cir }}$ of the basis current passing through nanoconstriction. The time $t$ dependence of the PNC resistance $R_{\mathrm{S}}$, within the window $0.2 \mathrm{~s}<t<100 \mathrm{~s}$, has been measured at zero external magnetic fields by a two-probe method using a homemade computer-controlled high sensitive electrical circuit. For each $R_{\mathrm{S}}$ vs. $t$ measurement, the magnetization of the nanocontacts is first saturated with a circular magnetic field $H_{\text {cir }}$ of the basis current $J$ (up to $10^{8} \mathrm{~A} / \mathrm{cm}^{2}$ ). After several minutes $J$ is turned to any $J / n$ value and the $R_{\mathrm{S}}(t)$ measurement starts. $H_{\text {cir }}$ generated from a uniform current density through a cylinder of radius $r_{0}$ is oriented along the azimuthal direction. As can be easily calculated from Ampère's law the radial dependence of the field is given by $B_{\phi}=2 \pi \times 10^{-5} \mathrm{Jr}$ for $0<r<r_{0}$ and $B_{\phi}=2 \pi \times 10^{-5} \mathrm{Jr}^{2} / r$ for $r>r_{0}$ $\left\{B[\mathrm{Oe}] ; \quad J\left[\mathrm{~A} / \mathrm{cm}^{2}\right] ; r[\mu \mathrm{m}]\right\}[9]$. According to the above procedure the measured time dependences $R_{\mathrm{S}}(t)$ of the PNC are thus given by the magnetic configuration in the vicinity of the nanocontact. The magnetic configuration is induced by a circular magnetic field of the current passing through nanoconstriction. This implies that the measurement probes only the narrow region close of the nanocontact. The parameters of nanocontact are determined by a mechanical way by the screw system. In order to investigate the relaxation behavior of these nanoconstrictions, the time-dependent resistance $R_{\mathrm{S}}$ measurements were performed at room temperature (RT), below $T_{\mathrm{C}}$ as shown in Fig. 1. The relaxation effects of $R_{\mathrm{S}}$ vs. $t$ displayed in Fig. 1, can be very well fitted by the two-term formula

$$
\begin{aligned}
& R_{\mathrm{S}}(t)=C+R_{0}\left[1-\exp \left(-\left(t / \tau_{1}\right)^{\beta}\right)\right] \\
& \quad+S_{\mathrm{R}} \ln \left(1+t / \tau_{2}\right) .
\end{aligned}
$$

We have found two distinctive regions of early and late

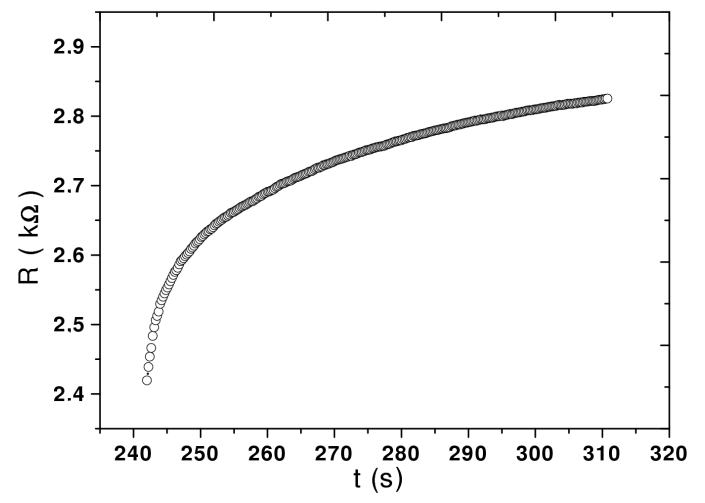

Fig. 1. The time dependence of the resistance $R_{\mathrm{S}}$ of LSMO PNC measured at RT. Solid line is the best fit, obtained by the phenomenological formula [1] described in the text.

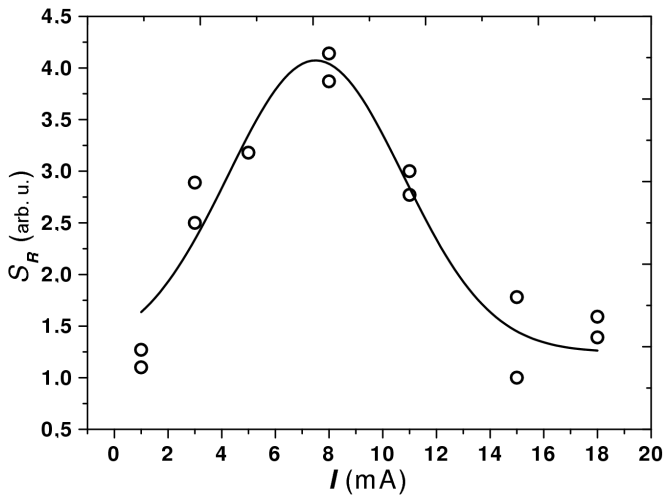

Fig. 2. Bell-shaped curve of the resistance viscosity $S_{\mathrm{R}}$ calculated from $R_{\mathrm{S}}(t)$ measurements at different values of the basis currents $I$.

stage of the resistance relaxation. In the early stage the relaxation is well fitted by a stretched exponential function with characteristic time $\tau_{1}$ and the late stage is well fitted by a logarithmic function with characteristic time $\tau_{2} . \quad S_{\mathrm{R}}$ denotes resistive viscosity. The appearance of both contributions evidences the existence of two sources of relaxation, which can be assigned to inhomogeneous changes of the angle between the magnetic moments of the neighboring $\mathrm{Mn}$ ions inside spin blocks and between these spin blocks. The resistance relaxation times $\tau_{1}$ and $\tau_{2}$ are of order of seconds and tens of seconds, respectively, and are dependent on the basis current flowing through the nanocontacts. These results point out the important role of structural arrangements of the Mn-ions clusters on the surfaces electrodes of the nanocontact. This could be explained by the assumption that the relevant magnetization controling the resistance is that of the surface and not the bulk (possibly because the carrier coherence length may be quite short compared to magnetic correlation length). We interpret the changes in resistance $R_{\mathrm{S}}$ as being due to the reorientation of the magnetization configuration in the surface of the PNC device caused by the rotational magnetic field $H_{\text {cir }}$ generated by the current passing through the device. Figure 2 shows resistance viscosity $S_{\mathrm{R}}$ vs. currents passing through the nanocontact during measurements of the relaxation effects obtained from fitting data $R_{\mathrm{S}}(t)$ with Eq. (1).

The passing currents create magnetic fields $H_{\text {cir }}$ of different values around coercivity field of the nanocontact. The surface resistance viscosity coefficient $S_{\mathrm{R}}$ versus the basis current exhibits a characteristic bell-shaped curve (Fig. 2). This form is known for the dependences of magnetic viscosities on magnetic field with the maximum near the coercivity [10]. The main message of the present experiments is that the relaxation of surface resistance $R_{\mathrm{S}}$ of the nanocontacts is characterized by two characteristics times, which is opposite to the bulk relaxation described by only one characteristic time [4]. The physical source of these results point out the important role of structural arrangements of the Mn-ions inside spin blocks 
and between the spin blocks or clusters on the surfaces of the nanocontact constrictions. The origin of this behavior is under investigation.

In conclusion, we have investigated the surface resistance relaxation of the nanocontacts fabricated from $\mathrm{La}_{0.7} \mathrm{Sr}_{0.3} \mathrm{MnO}_{3}$ perovskites. Electrical transport through the nanocontact surfaces PNC provides another, though indirect, access to surfaces magnetic order, if the microscopic relation between magnetism and transport in the PNC is known. In addition, the possibility of measuring relaxation effects through transport measurements removes the need of a direct surfaces magnetization measurement, which is difficult for nanoscale magnets.

\section{References}

[1] E. Dagatto, T. Hotta, A. Moreo, Phys. Rep. 344, 1 (2001).
[2] F.J. Wang, C.G. Yang, Z. Valy Vardeny, X.G. Li, Phys. Rev. B 75, 245324 (2007).

[3] C. Zener, Phys. Rev. 82, 403 (1951).

[4] M. Sirena, L.B. Stern, J. Guimpel, Phys. Rev. B 64, 104409 (2001).

[5] R. van Helmolt, J. Wecker, T. Lorenz, K. Samwer, Appl. Phys. Lett. 67, 2093 (1995).

[6] H. Dulli, P.A. Dowben, S.-H. Liou, E.W. Plummer, Phys. Rev. B 62, R14629 (2000).

[7] J. Moreland, J.W. Ekin, J. Appl. Phys. 58, 3888 (1985).

[8] C.J. Muller, J.M. van Ruitenbeek, L.J. de Jongh, Physica C 191, 485 (1992).

[9] K. Bussmann, G.A. Prinz, S.-F. Cheng, D. Wang, Appl. Phys. Lett. 75, 2476 (1999).

[10] I.D. Mayergoyz, A. Adly, C. Korman, Mingwei Huang, C. Krafft, J. Appl. Phys. 85, 4358 (1999). 